\title{
Identification of Material Models of Nanocoatings System Using the Metamodeling Approach
}

\author{
Magdalena Kopernik, Andrzej Stanisławczyk, Jan Kusiak, and Maciej Pietrzyk \\ AGH University of Science and Technology, Mickiewicza 30, 30-059 Kraków, Poland \\ magdalenakopernik@interia.pl, astan@agh.edu.pl, \\ kusiak@agh.edu.pl, pietrzyk@agh.edu.pl
}

\begin{abstract}
Hard systems of nanocoatings deposited using PVD (physical vapor deposition) are used in the artificial heart prosthesis. Correct determination of nanomaterial parameters is crucial for accuracy of simulation. The objective of this work is identification of material parameters of nanocoatings in hard system using the inverse analysis based on the artificial neural network metamodeling. The inverse analysis was preceded by the development of the Finite Element Method (FEM) model dedicated to the nanoindentation test of the hard nanocoatings system. The performed sensitivity analysis is focused on determination of parameters, having the highest influence on FEM model response. The obtained, reliable FEM model was used next in the inverse analysis. The objective of that analysis was evaluation of the parameters of the individual layers of the nanocoating system. In order to decrease the computation time connected with the inverse analysis, the metamodeling approach was proposed. The used metamodel was based on the artificial neural network technique. The obtained results confirm the usefulness of the presented method in the identification of the material properties of the complex, nanocoating systems.
\end{abstract}

\section{Introduction}

Thin hard nanocoating systems exhibit interesting tribological and functional properties, which are difficult to achieve in conventional, homogenous materials. On the other hand, due to very small scale and contrasting physical properties in adjacent, very thin layers, physical and numerical modeling of these systems face essential difficulties. Hard nanocoatings and their systems are usually investigated in experimental nanoindentation tests, because other, standard experimental methods performed in macro and micro scale are not suitable for such case [12]. Analytical methods for nanoindentation tests, which lead to evaluation of mechanical properties, were developed by Oliver and Pharr [12]. However, all these solutions are dedicated to monolayer materials. Therefore, the authors of the present work have undertaken some attempts towards the numerical FEM modeling of multilayer system [7]. FEM modeling of nanoindentation test appears difficult, because of the small thickness of layers, which involves necessity of mesh regeneration. Accuracy of the FEM simulation of the layered, multimaterial system depends on adequate evaluation of the properties of every single layer, which is crucial in modeling of nanocoatings. As the result of mentioned above difficulties, the

A. Korytowski et al. (Eds.): System Modeling and Optimization, IFIP AICT 312, pp. 319-330, 2009.

(C) IFIP International Federation for Information Processing 2009 
direct numerical model for nanoindentation test is computationally expensive. Therefore, development of the alternative, computationally effective method, based on the metamodel principle, is the main objective of the present work.

The first part of work describes the nanoindentation test of hard nanocoatings and explains how hardness in nanoscale is measured. The next part of the paper is dedicated to the development of the efficient and robust FEM model of nanoindentation test. FEM modeling was preceded by the sensitivity analysis oriented towards the determination of material model parameters and nanotest settings, which have the greatest influence on a response of generated FEM model of nanocoatings system.

The main objective of the present work is the inverse analysis, which allows the identification of material parameters of inner nanocoating in system of hard nanocoatings composed of various nanomaterial layers. The metamodel approach [9] based on the FEM modeling and artificial neural network techniques [6]10] is proposed in the paper.

\section{Nanoindentation Tests}

The first objective of the work was investigation of properties of tribological hard nanocoatings system, which is composed of TiAlN [3] and TiN [2]. These materials are deposited on the elastic substrate like carbide using PVD technique. Titanium nitride is used for some particular and the most demanding applications, because it increases the biocompatibility of the material. An artificial left blood chamber and its constructional element, which is an aortic valve, are the good examples of biotechnological application [11] of these materials, especially of TiN. The properties (hardness and Young's modulus) of a specimen are examined in the experimental nanoindentation tests.

\subsection{Examined Material}

The specimen (technical material) of titanium nitride basis and thin mixed hard elasticplastic nanolayers deposited on elastic substrate was investigated. The material system of eleven PVD, thin material layers on carbide (infinite thickness) is shown in Fig. 1 Two different coatings are deposited periodically. Coating 2 (TiN, an elastic material) is $40 \mathrm{~nm}$ thick and is repeated three times. Coating 1 (TiAlN, an elasticplastic material) is $400 \mathrm{~nm}$ thick and is repeated four times.

\subsection{Experiment}

The objective of the nanoindentation test is to evaluate the mechanical properties of indented material like hardness and Young's modulus. The experimental nanoindentation test is performed in load or depth controlled mode using a Nano Test System [3]2]. Diamond $(E=1141 \mathrm{MPa}, v=0.07$ ), Berkovich pyramid (tip radius $R=150 \mathrm{~nm}$, pyramid angle $\alpha=70.32^{\circ}$ ) penetrates into the specimen. The schematic illustration of experiment and the top view of Berkovich indent is shown in Fig. 2.

The multistage process of deformation in nanoindentation test is performed in the case of testing the multicoating material. This procedure is necessary for specimen composed of nanocoatings to eliminate the effect of scatter in results and to create a 


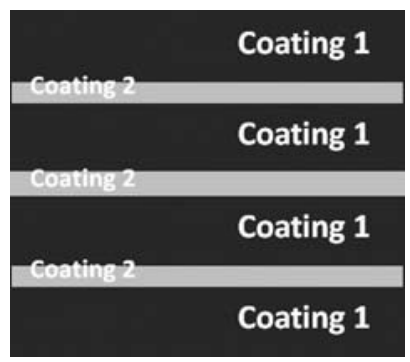

Fig. 1. Analyzed system of nanocoatings

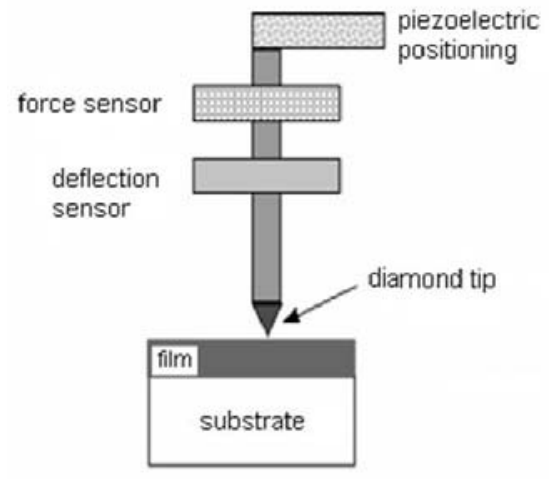

(a)

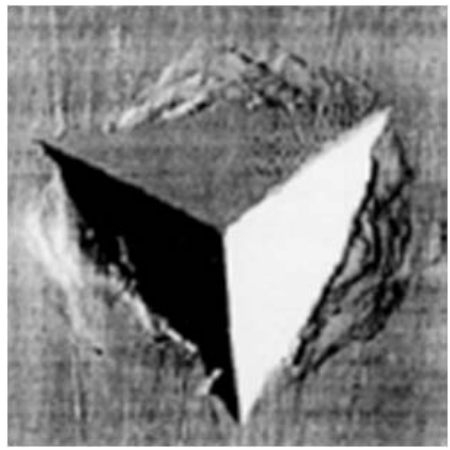

(b)

Fig. 2. (a) Schematic illustration of nanoindentation test [5], (b) The real view of Berkovich indent [5]

possibility to achieve the response of bottom layers during long term deformation process. The indentation test supplies force versus indentation depth (tool displacement) data. The load is the main output from the experiment and the Martens hardness for deformed material is calculated on the basis of force/displacement or depth data [12]3|2].

\section{FEM Model}

The earlier research of the authors [7|9 10]8] is focused mainly on overcoming numerical difficulties occurring in FEM simulation of deformation process of hard nanocoatings, caused by the nanothickness of layers, necessity of remeshing and scaling operations, as well as the multimaterial, multistage character of simulation and efforts to decrease the computing costs.

The objective of the present work was the development of FEM model of nanoindentation test accounting for different control parameters of the test, like indenter shape and friction conditions, as well as sensitivity of the response of the specimen with respect to material model parameters. The selected results, which are crucial for the development of efficient FEM model of nanoindentation, are presented below. 


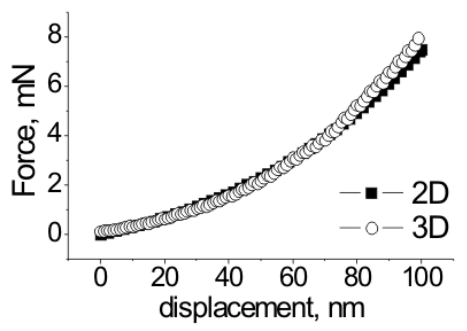

Fig. 3. Force versus depth results for 2D FEM axisymmetric and 3D FEM models [8]

To decrease the computing costs, the simplified 2D axisymmetric model of the nanoindentation test was considered and the Berkovich indenter was treated as a conical one. Such simplified model does not cause a loss of important information, which was validated through the full 3D FEM simulation of nanoindentation tests [1.4]. The results of comparison of 2D simulation and the full 3D model, obtained by the authors of the present work are shown in Fig. 3] [8]. They confirm that simplified 2D model can be used in the further research of the present work, which allows decreasing the computation costs. The velocity of the indenter [3/2] in experimental nanoindentation test is constant and very small. According to the experimental procedure, the value of the indenter constant velocity does not have an effect on behavior of specimen's material and in each simulation is equal to $1 \mathrm{~nm} / \mathrm{s}$.

\subsection{Sensitivity to Indenter Shape, Friction and Material Model Parameters}

The design of conditions of an effective nanoindentation test, as well as adequate choice of the FEM model parameters, were preceded by the sensitivity analysis. This method allows the estimation of the influence of the individual process parameters on the value of the analyzed one. The considered parameter was the total load (force) of the nanoindentation test, therefore, its sensitivity with respect to the process parameters was determined. The performed sensitivity analysis was based on the finite difference approximation. The sensitivity coefficients $\varphi_{p_{j}}$ were defined as:

$$
\left.\varphi_{p_{j}}\right|_{\mathbf{p}^{*}}:=\left.\frac{p_{j}^{*}}{F_{a v}\left(\mathbf{p}^{*}\right)} \frac{\partial F_{a v}}{\partial p_{j}}\right|_{\mathbf{p}^{*}} \cong \frac{p_{j}^{*}}{F_{a v}\left(\mathbf{p}^{*}\right)} \frac{F_{a v}\left(\mathbf{p}^{*}+\Delta p_{j} \mathbf{e}_{\mathbf{j}}\right)-F_{a v}\left(\mathbf{p}^{*}\right)}{\Delta p_{j}}
$$

where: $\mathbf{p}^{*}=(R, \alpha, \mu, E, K, n)$ - vector composed of considered parameters, $\mathbf{e}_{\mathbf{j}}$ - vector of the canonical basis, $\Delta p$ - variation of the parameter $p, F_{a v}$ - average value of the total load, calculated as follows:

$$
F_{a v}=\frac{1}{t} \int_{0}^{t} F(\tau) d \tau
$$

where: $F(\tau)$ - the load at the time $\tau, t$ - total time of the process.

The sensitivities of the total load of the nanoindentation test with respect to the indenter shape parameters $(R, \alpha)$, friction coefficient $(\mu)$ and specimen's material parameters 


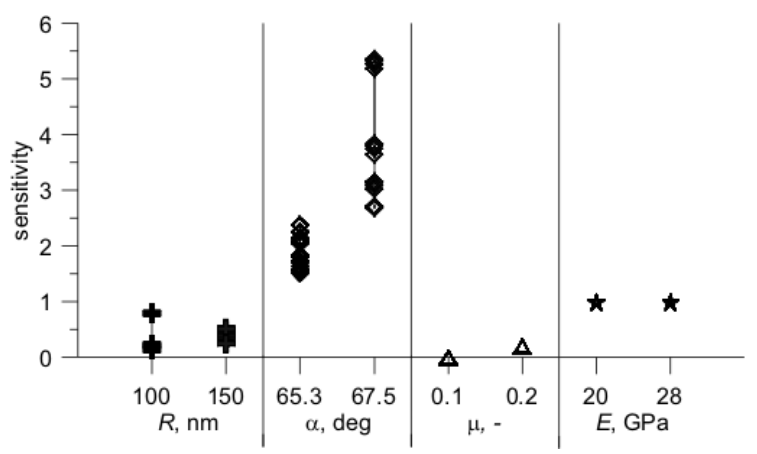

(a)

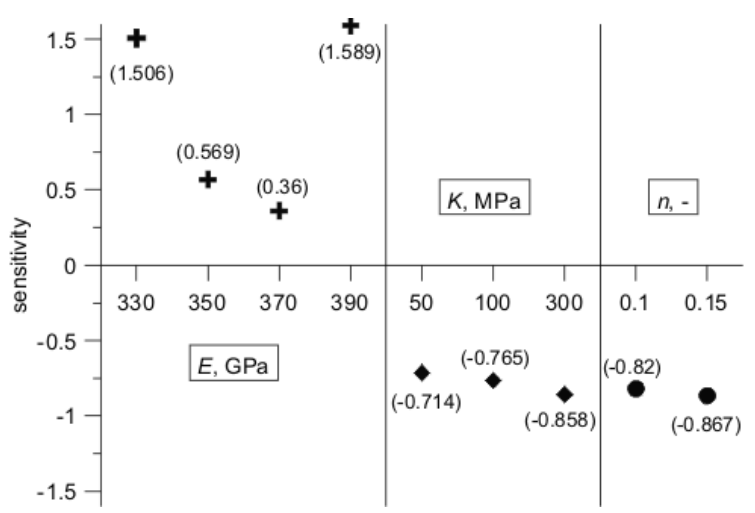

(b)

Fig. 4. (a) Sensitivity of the load with respect to geometrical indenter tip parameters: $R, \alpha$ and friction coefficient $\mu$ for monocoating specimen. (b) Sensitivity of the load with respect to the material model parameters $E, K$ and $n$ for the specimen composed of 3 hard nanocoatings.

$(E, K, n)$ were analyzed. Two different specimens were examined: an elastic monocoating specimen - $400 \mathrm{~nm}$ thick and a specimen composed of 3 hard nanocoatings.

For the first, monocoating specimen, twelve Berkovich indenters with four tip radii equal to $100,110,150$ and $160 \mathrm{~nm}$, as well as three tip vertex angles: 65.3, 67.5 and $70.32^{\circ}$, all with round tip were investigated. The Coulomb friction law was assumed with the following Coulomb friction coefficient values: $\mu=0.1, \mu=0.15, \mu=0.2$ and $\mu=0.25$. The Poisson ratio $v=0.177$ and four elastic moduli: $E_{1}=20, E_{2}=22, E_{3}=28$, and $E_{4}=30 \mathrm{GPa}$ were used in the material model defined by:

$$
\sigma=E \varepsilon
$$

where: $\sigma$ - work-hardening stress, $\varepsilon$ - strain, $E$ - Young's modulus.

196 FEM simulations were performed. The obtained results of the sensitivity analysis presented in Fig. $4 \mathrm{a}$ indicate that the nanoindentation test is the most sensitive to the geometrical parameters of the tip, especially to the higher values of the tip angle. The 
friction does not require special consideration, because very low values of sensitivity coefficients are observed and, therefore, it can be omitted in future analysis.

The second examined specimen had the following 3 hard nanocoatings: coating 1 (elastic, $400 \mathrm{~nm}$ thick) was repeated twice and coating 2 (elasticplastic, $40 \mathrm{~nm}$ thick) was a single interlayer. The coating 1 was considered as an elastic material defined by Eq. 3. The elasticplastic material of the coating 2 was described by the following relationship:

$$
\sigma=K \varepsilon^{n}
$$

where: $\sigma$ - work-hardening stress, $\varepsilon$ - strain, $K$ - hardening coefficient, $n$ - hardening exponent.

The considered values of the Young's modulus of the material model Eq. 3 of the coating 1 were: $E=330,350,370,390$ and $410 \mathrm{GPa}$. The chosen values of parameters in material model Eq. 4 of the coating 2 were: $K=50,60,100,110,300$ and $310 \mathrm{MPa}$, while $n=0.1,0.15,0.2$ and 0.25. Diamond, Berkovich indenter (radius $R=150 \mathrm{~nm}$ and pyramid angle $\alpha=70.32^{\circ}$ ) penetrates into specimen. The friction coefficient $\mu$ is assumed 0. Finally, 144 FEM simulations were performed.

The obtained results of sensitivity calculations for the second, multicoating specimen are presented in Fig.4b. They show that for chosen material models Eq. 3 and Eq. 4, the load is the most sensitive to the parameters $E$ and $n$, as well as to the parameter $K$. It means that each parameter of the material models Eqs. 3 and 4 is important and has to be considered in future FEM models of nanoindentation test.

\subsection{The Final FEM Model of Nanoindentation Test}

The aim of research described in Sect. 3.1 was the analysis of the influence of the deformation process and material model parameters on the total load (force), as well as on the evaluation of optimal conditions and input settings for FEM model of nanoindentation test of the hard nanocoatings system. The defined process and material model parameters used in the developed FEM model of nanoindentation test are:

- the angle of indenter $\alpha=70.32^{\circ}$ and tip radius $R=150 \mathrm{~nm}$,

- the indenter velocity $v=1 \mathrm{~nm} / \mathrm{s}$ and final displacement $d=100 \mathrm{~nm}$,

- the parameters $E, K, n$ in used material models Eqs. 3 and 4, which are specified in the last section of Sect. 3.1 and presented in Fig. $4 \mathrm{~b}$,

- the specimen has three coatings with material models are described by Eqs. 3 and 4 respectively for elastic coating 1 and elasticplastic coating 2,

- the frictionless conditions between indenter and specimen $(\mu=0)$.

The final, used in further calculations of the present work, FEM mesh has 7000 nodes and 13000 elements (Fig. 57).

The described FEM model was implemented into the FORGE 2 code. The example of equivalent strain distribution is plotted in Fig. 5b. It can be seen that the maximum of strain is located in the inner coating. 


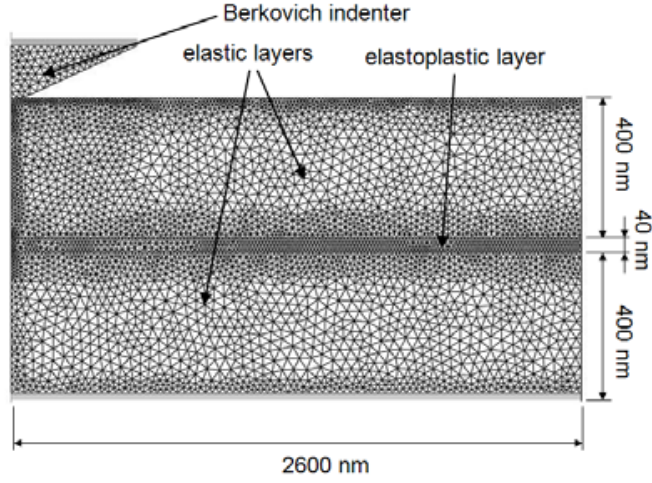

(a)

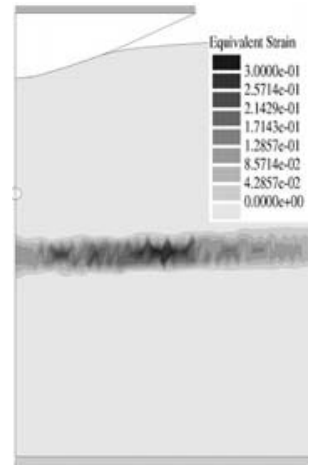

(b)

Fig. 5. (a) The developed FEM model (specimen and indenter) of the nanoindentation test. (b) Distributions of the equivalent strain of 3 hard nanocoatings.

\section{Inverse Analysis with Metamodel}

Generally, the main goal of the inverse analysis is evaluation of the real parameters of the model for the tested material. The aim of the present work is evaluation of these parameters on the basis of the nanoindentation test for multi-nanocoating systems. The known and described widely in [12] analytical methods used in experimental nanoindentation test lead to evaluation of mechanical properties (hardness, Young's modulus) and they produce desired results, but only for monolayer specimen. It is impossible to extrapolate these solutions to the multilayered nanocoatings.

Therefore, the objective of this research is to evaluate the properties of the inner layers of the multinanocoating system. The inverse analysis was suggested by the authors to solve this problem. The load measured for the whole nanocoatings system is the main output from the nanoindentation experiment, which is indirectly used in the goal function of the classical inverse approach. Since the inverse analysis of such complex nanomaterial system is very time-consuming procedure, the classical inverse analysis was coupled with the artificial neural network (ANN). The ANN approach allows significant reduction of the computational costs.

\subsection{Classical Inverse Approach}

The identification of material model can be done using the classical inverse approach. The objective of the inverse analysis is to find, using the optimization procedure, the material model parameters, which give the best matching between results of the FEM simulation and the experiment. The discrepancy between these values is the optimization goal function, which has to be minimized.

Unfortunately, in many cases, the evaluation of goal function requires numerous time-consuming FEM simulations. It makes the computation time of the whole inverse 
analysis unacceptable. In the analyzed problem one FEM simulation of nanoindentation test, for conditions described above, is computed at least about three hours and the whole inverse analysis for one simulation of experiment may last many days.

\subsection{Metamodel and Results}

The inverse method can be speeded up by using the fast metamodeling approach, instead of running thousands FEM simulations. The idea of the metamodel approach can be briefly defined as modeling of the existing model. Usually, in the metamodeling procedure, various methods of approximation or artificial intelligence tools are used to modeling of existing models of analyzed processes. The latter approach is applied in the present work.

For the purpose of the inverse analysis of considered nanoindentation test the proposed metamodel is based on the artificial neural network models. Obviously, the ANN metamodel creation demands numerous time-consuming FEM simulations as the input data. But this is done once, and later on, the whole inverse procedure may be performed fast for many simulations of experiment. The idea of the metamodel creation and its application in the inverse analysis are shown in Fig. 6 .

As it was mentioned, the metamodel of the FEM output data of the nanoindentation test, obtained in the FORGE 2 simulations, is based on the artificial neural network approach. The Multi Layer Perceptron (MLP) of the 4-2-1 architecture is used (logistic transfer functions in the first and second layers; linear activation function in the output layer - see Fig. 7).

The ANN input data are the parameters $E, K$ and $n$ of the material models Eqs. 3 and 4 of the analyzed multinanocoating specimen, as well as the indenter displacement $d$. The ANN output data corresponds to force $F$.

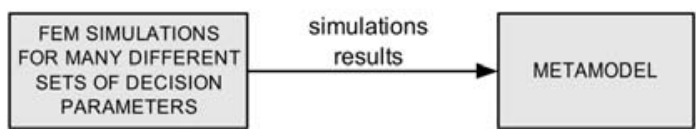

(a)

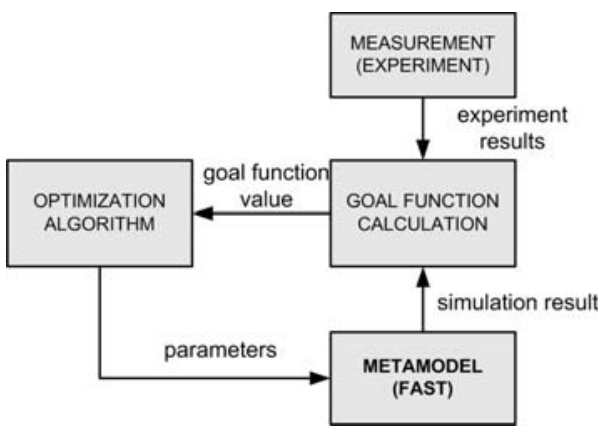

(b)

Fig. 6. The metamodel: (a) creation, (b) application in the inverse analysis 


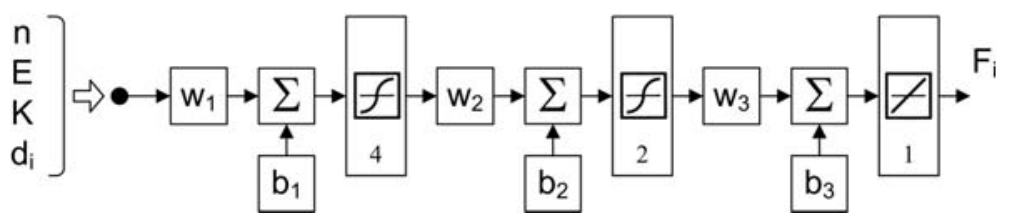

Fig. 7. The metamodel based on the ANN

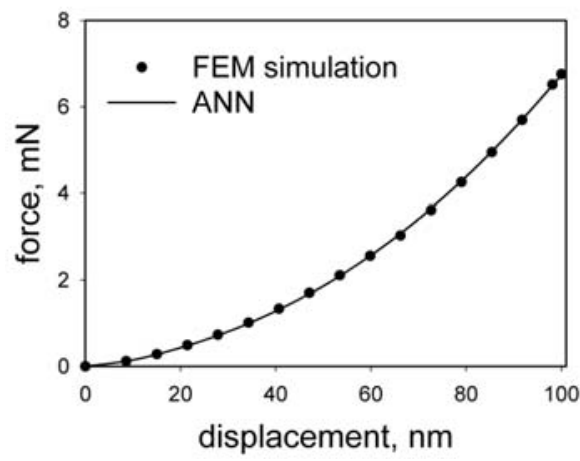

Fig. 8. Results of the artificial neural network test for work-hardening curves of multinanocoating specimen

144 data sets for various $n, K$ and $E$ were used. Each set was composed of 25 values of force versus displacement data. 142 sets of data were training data, and two sets were used as for ANN test. The network was trained using Levenberg-Marquardt algorithm [6]. The network was tested for $n=0.15, E=370 \mathrm{GPa}, K=100 \mathrm{MPa}$ and the results are shown in Fig. 8 . Root mean square error for the two test sets is equal to $20 \mu \mathrm{N}$, which confirms good predictive capability of the network.

The trained network was used next as the metamodel in the inverse analysis. The analyzed goal function of the inverse problem was the root mean square error between experimental data and the output of the network:

$$
\phi(n, E, K)=\sqrt{\frac{1}{N} \sum_{i=1}^{N}\left(F_{E X P}(i)-F_{A N N}\left(n, E, K, d_{i}\right)\right)^{2}}
$$

where: $F_{E X P}$ - force vs displacements simulated by FEM, $F_{A N N}$ - ANN predicted values of the force, $d_{i}$ - displacements, $N$ - number of computing steps.

To find the minimum value of the goal function (Eq. 5] the hybrid optimization procedure was applied. The genetic algorithm was used in the first phase to the localization of the minimum, while the Quasi-Newton algorithm was used in the final search. The whole algorithm of the inverse method with metamodel is shown in Fig.9.

The experimental data was generated by FEM simulation for the set of material model parameters: $n=0.175, E=400 \mathrm{GPa}$ and $K=270 \mathrm{MPa}$. The results for examined case are presented in Fig. 10. Evaluated minimum of the goal function (Eq. 5) is 


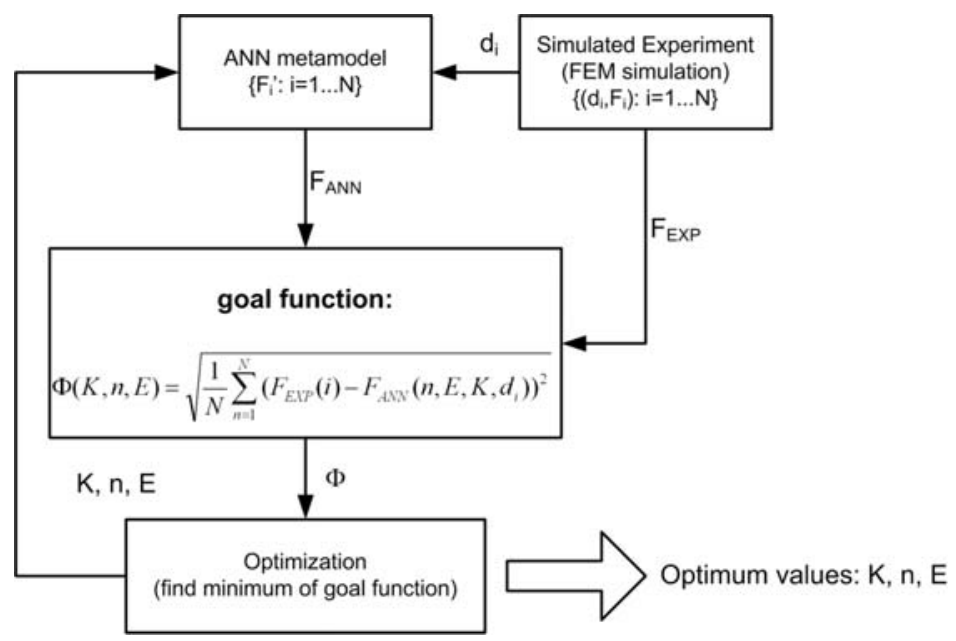

Fig. 9. Algorithm of the inverse analysis with metamodel

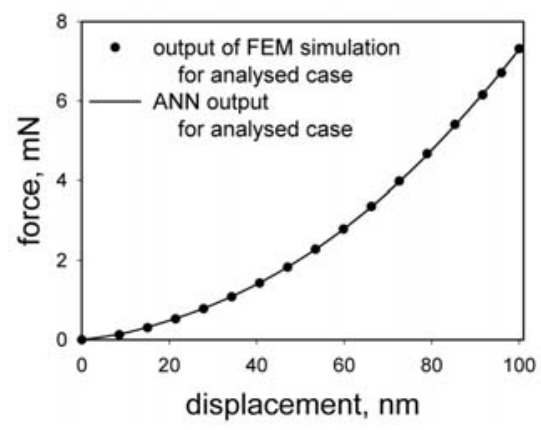

Fig. 10. Results of the inverse analysis for the analyzed case

found at $n=0.17, E=397 \mathrm{GPa}$ and $K=331 \mathrm{MPa}$ for analyzed case. The goal function value is $\phi=21 \mu \mathrm{N}$.

It is shown in Fig. 10 that the experimental (simulated) points match very well the found solution. Unfortunately, the problem is irreversible. It means that for one set of force versus displacement many different solutions can be found. This statement is also confirmed by the second plot, which is shown in Fig. 11. This plot presents logarithm of goal function (Eq. 5) for the examined case. The chosen goal function for simulated experimental data set takes minimal values in some area located around experimental parameters. The minimum of goal function is shallow and therefore, the optimization result depends on the starting point. The ambiguity problem will be greater for the real experimental data (not simulated), because there is a big scatter in experimental results. Thus, the future form of used material model (Eq. 4) should be modified by adding more parameters or chosen the more complex material model. 


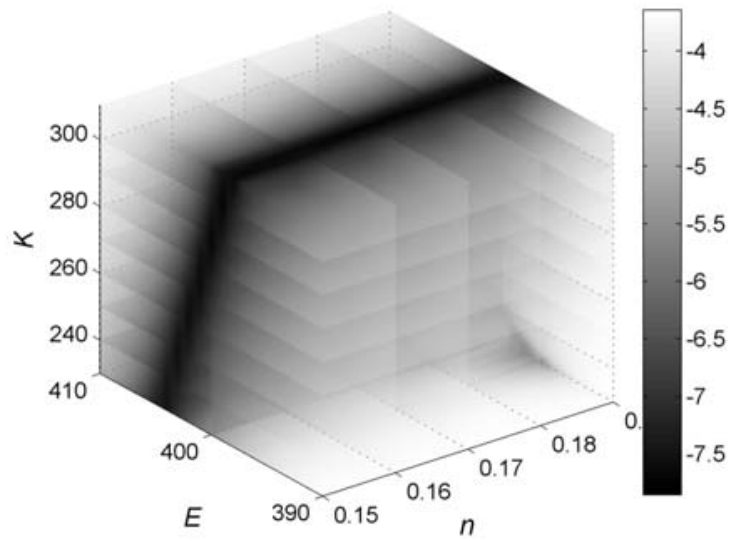

Fig. 11. Plot of the logarithm of the goal function for the experimental data set

\section{Conclusions}

The presented research and reached results lead to the following conclusions:

- Presented metamodeling approach is useful for considered optimization problem when the evaluation of the goal function is time-consuming. The proposed algorithm allows radical decrease of the number of long-term FEM calculations. Despite of the initial computational efforts connected with the ANN training, the final use of the network as the metamodel in the evaluation of material model parameters lasts only a few seconds. So, the reached time-profit is very high.

- The quality of results is very good. Trained ANN gives good compatibility with the test set.

- There is a certain disadvantage of presented approach - a weak ambiguity, because similar output curves can be obtained for different combination of chosen input material model parameters. This observation is proved by the plot of the used goal function (Eq.5), which is shown in Fig. 11 The goal function takes minimal values in some area located around chosen experimental parameters. The minimum of goal function is shallow and vast. Therefore, the analyzed problem is irreversible. The disadvantage of such approach appears, because it is impossible to find out precisely, which combination of parameters in material model is the best solution of the inverse problem. Therefore, the future research of the authors will be focused on solving the uniqueness problem by modification of the form of material model, by adding more parameters or choosing a more complex material model.

- The ANN based metamodel can be used together with another optimization procedure, for example the one defined by heuristic algorithms.

- The key aspects of all the prospects and conclusions are important from the point of view of the authors, because the examined system of nanocoatings will be used for demanding biomedical application. These coatings will be also deposited on polyurethane by PLD (Pulsed Laser Deposition) technique and are supposed to be 
used for artificial heart prosthesis as the constructional materials. Numerical model of such artificial organ needs the exact material model parameters of all its material layers.

Acknowledgements. Financial assistance of the MNiSzW, project no. N507 136 32/ 3962 , is acknowledged.

\section{References}

1. Albrecht, H.J., Hannach, T., Hase, S., et al.: Nanoindentation: a suitable tool to determine local mechanical properties in microelectronic packages and materials? Arch. Appl. Mech. 74, 728-738 (2005)

2. Beake, B.D., Ranganathan, N.: An investigation of the nanoindentation and nano/microtribological behaviour of monolayer, bilayer and trilayer coatings on cemented carbide. Mat. Sci. Eng. A23, 46-51 (2006)

3. Beake, B.D., Smith, J.F., Gray, A., et al.: Investigating the correlation between nano-impact fracture resistance and hardness/modulus ratio from nanoindentation at 25-500 $\mathrm{C}$ and the fracture resistance and lifetime of cutting tools with Ti1-xAlxN ( $\mathrm{x}=0.5$ and 0.67 ) PVD coatings in milling operations. Surface and Coatings 201, 4585-4593 (2007)

4. Chollacoop, N., Dao, M., Suresh, S.: Depth-sensing instrumented indentation with dual sharp indenters. Acta Mater 51, 3713-3729 (2003)

5. Fischer-Cripps, A.C.: Nanoindentation. Springer, New York (2004)

6. Koker, R., Altincock, N., Demir, A.: Neural network based prediction of mechanical properties of particulate reinforced metal matrix composites using various training algorithms. Materials and Design 28, 616-627 (2007)

7. Kopernik, M., Pietrzyk, M.: 2D numerical simulation of elasto-plastic deformation of thin hard coating systems in deep nanoindentation test with sharp indenter. Archives of Metallurgy and Materials 52, 299-310 (2007)

8. Kopernik, M., Szeliga, D.: Modelling of nanomaterials - sensitivity analysis to determine the nanoindentation test parameters. Computer Methods in Materials Science 7, 255-261 (2007)

9. Kopernik, M., Stanisławczyk, A., Kusiak, J., et al.: Identification of material models in hard system of nanocoatings using metamodel. In: Korytowski, A., Mitkowski, W., Szymkat, M. (eds.) Abstr., 23rd IFIP TC7 Conference on System Modelling and Optimization, Kraków (2007)

10. Kopernik, M., Stanisławczyk, A., Szeliga, D.: Problems of material models of hard nanocoatings. In: Dems, K. (ed.) Proc. 17th Int. CMM Conf., Łódź-Spała (2007)

11. Kustosz, R., Major, R., Wierzchoń, T., et al.: Designing a new heart. Academia 3, 14-17 (2004)

12. Oliver, C., Pharr, G.M.: An improved technique for determining hardness and elastic modulus using load and displacement sensing indentation experiment. J. Mater. Res. 7, 1564-1583 (1992) 